\title{
Nipple-areola complex macro photography to test skin hydration and cracked nipples in breastfeeding women
}

\author{
Vincenzo Zanardo*, Francesca Volpe, Lara Giliberti, and Gianluca Straface \\ Division of Perinatal Medicine, Policlinico Abano Terme, Abano Terme, Italy
}

\begin{abstract}
Background: Appropriate hydration is fundamental in preventing nipple-areola skin barrier damage and cracking nipples.

Methods: Nipple-areola complex skin hydration status with contemporary macro photography (x100) was evaluated at $36 \pm 6$ postpartum hours in breastfeeding women using non-invasive bioengineering methods.

Results: Macro photography was found to be effective in describing both stratum corneum hydration features of breast nipple-areola complex and cracking nipples.

Conclusion: A non-invasive device made it possible to test nipple-areola complex hydration and to asses by macro photography the skin effects of the hydration and of cracking nipples during the most critical phase at the initiation of breastfeeding.
\end{abstract}

\section{Introduction}

The human female's nipple-areola complex (NAC) is of particular interest towards its crucial role in the natural progression of the birth to breastfeeding continuum [1]. In newborn mammals the nipples provide access to colostrum and milk facilitating early breast-feeding attempts and successful nipple grasping and feeding [1-4].

When newborns latch on to the breast, mothers often experience pain which is caused by all or parts of the nipple being pulled into the baby's mouth [5]. The first days after birth are often characterized by breast discomfort due to rough treatment of the tender area. In fact, sore cracked nipples are an early common problem into breastfeeding, with an incidence ranging from $11 \%$ to $96 \%$, and can be so severe as to lead to extreme nipple pain and premature weaning $[5,6]$.

One of the most common causes of damaged nipples is NAC dryness and this can usually be corrected if the suggestions of breastfeeding experts are followed [7]. Many women, however, delay seeking specific treatment until substantial damage has already occurred and this can delay the healing process [8]. The NAC is repeatedly exposed to the rough treatment and wet environment of the infant's mouth; after feedings mothers may not use the best hydration technique to protect nipple' skin barrier, and mother's clothing between feedings may not permit sustained hydration. In addition, traumatized nipples, exposed not only to maternal skin flora but also to the infant's oral flora, readily become infected by bacteria or yeast infections which themselves can delay and impede healing [9].

The surface of the skin is covered with a protective acidic hydrolipid film with $\mathrm{pH}$ 4.5-5.5 which is an emulsion of substances dissolved in water, composed of sebaceous and sweat gland secretion and products of decomposed corneocytes, whose bactericidal and fungicidal properties are based on mild acidity [10-12]. This emulsion also prevents evaporation of water from the surface of the skin, thus contributing to the maintenance of stratum corneum hydration [13].

Appropriate areola hydration is a key factor in preventing and treating sore cracked nipples, mastitis, and breast abscesses secondary to skin barrier damage [14]. Moreover, the water content of the stratum corneum and skin surface lipids form a balance which is very important for skin function and integrity [14]. These mechanisms of action are, however, not entirely clear and until now they have not been extensively studied.

Therefore, the aim of this non-invasive investigation was to asses by macro photography the hydration pattern of the nipple-areola complex skin and of cracking nipples during the most critical phase at the beginning of breastfeeding. The secondary aim of the study was to test related physiological characteristics of areola skin, hydration and $\mathrm{pH}$.

\section{Methods}

During a recent longitudinal prospective study to evaluate the dermal effects of Emu oil on areola skin hydration soon after birth in breastfeeding women [15], we planned additional analyses to determine if nipple-areola complex hydration and cracking nipples, are associated with a macro photography pattern. This prospective, open-label, controlled clinical trial was carried out from January $1^{\text {st }}$ to February $28^{\text {th }}, 2014$ at the Policlinico Abano Terme. It was certified by the National Observatory on Women's Health (O.N.D.A.), an association that raise awareness of the importance of preventing and caring for illnesses afflicting women.

Early after delivery, 50 at term breastfeeding puerperae took part in the trial focused on the hydrating properties of an Emu oil-based cream [15]. Institutional Review Board (Policlinico Abano Terme, Abano

Correspondence to: Prof. Vincenzo Zanardo, Division of Perinatal Medicine, Policlinico Abano Terme, Piazza Colombo 1, 35031 Abano Terme, Italy, Tel: +39 049 720027, E-mail: vincenzo.zanardo@libero.it

Received: January 22, 2016; Accepted: February 05, 2016; Published: February 08,2016 
Terme, Italy) approval was obtained before the study began.

The study was carried out within the hospital's ordinary care protocol after maternal informed consent. In accordance with the hospital's standard practice, following an uneventful delivery, infants are placed on the mother's chest for about 15 min during which time the midwife assists in the first suckling episode. Infants are then dried, receive umbilical care, and are weighed before their first bath. During the subsequent 2 days in our ward, new-borns room-in with their mothers, which are encouraged to insist on feeding them (with not more than 3-hr inter-feeding intervals).

During feeding breaks, the areolae were assessed by a single physician (VZ) using non-invasive bioengineering methods, the Soft Plus 5.5 (Callegari SPA, Parma, Italy) corneometer, which measures electrical capacitance of the skin surface expressed in conventional units (c.u). It was used to assess hydration values, $\mathrm{pH}, \mathrm{T}^{\circ}$, elasticity, and macro photography (x100) [16].

Testing and macro photography were performed at a fixed point, defined as areolar zone corresponding to the superior internal quadrant skin of both breasts, as previously reported [15].

\section{Results}

Because of median (Interquartile Range, IQR) study population hydration was 60.0 (44.8-73.0) conventional units, c.u., and the hydration values were normally distributed, we chose to report the 4 breastfeeding women values representative of lower, median, higher hydration and of cracking nipples. Data were revealed by hydration and $\mathrm{pH}$ skin barrier measure and by dermatological features obtained by macro photography (Table 1 ).

Areola at highest hydration quartile has a smooth skin aspect striped like a skein of cotton candy. The present puerpera has associated a hydration of $76 \%$ and a $\mathrm{pH}$ of 4.2 (Figure 1).

Areola at median hydration levels has a skin that appears as bright parallel stripes. The present puerperal has associated a hydration of $60 \%$ and a $\mathrm{pH}$ of 4.1 (Figure 2).

Areola at lowest hydration quartile has a paved skin with heavy crossing stripes which looks like snakeskin. The present puerperal has associated a hydration of $39 \%$ and a pH of 4.0 (Figure 3).

Cracking nipples appear as a paved skin with heavy, bleeding crossing stripes. The present puerperal has associated a hydration of $34 \%$ and a $\mathrm{pH}$ of 4.6 (Figure 4 ).

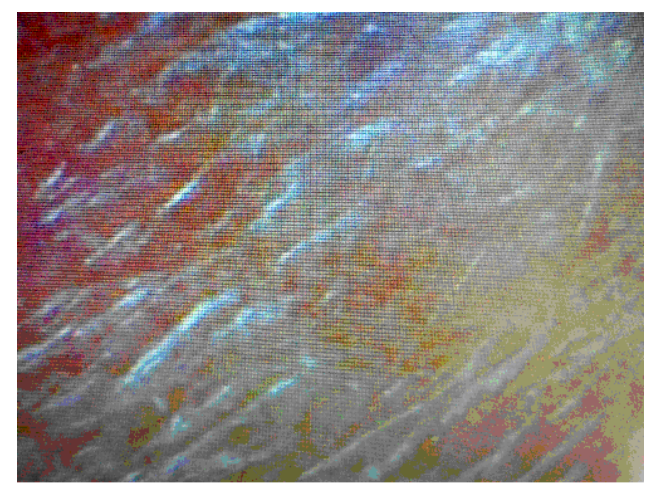

Figure 1. HIGH HYDRATION: aspect like a skein of cotton candy. Hydration: 76; $\mathrm{pH}$ : $4.2 ; \mathrm{t}^{\circ}: 34.4$; Elasticity: 41

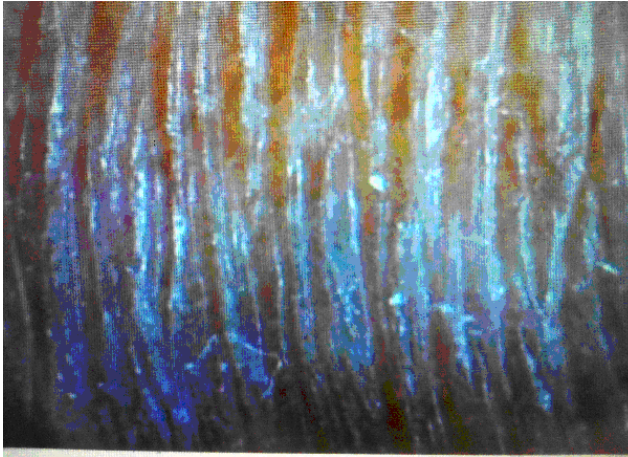

Figure 2. MEDIAN HYDRATION: skin that appears as bright parallel stripes. Hydration: 60; $\mathrm{pH}: 4.1 ; \mathrm{t}^{\circ}$ : 34.9; Elasticity: 35 .

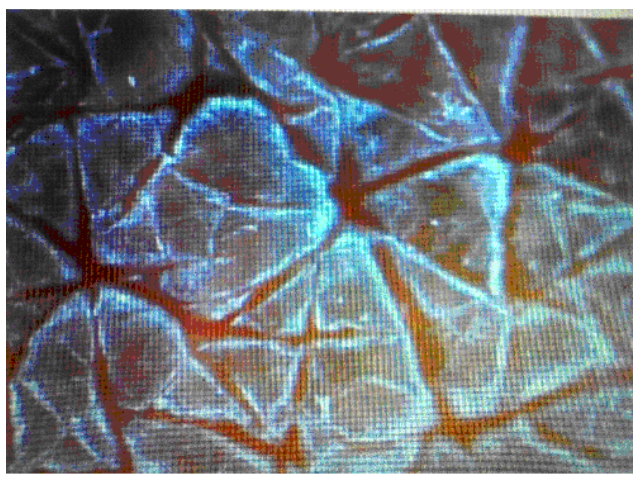

Figure 3. LOW HYDRATION: aspect like heavy crossing stripes which looks like snakeskin. Hydration: $39 ; \mathrm{pH}: 4.0 ; \mathrm{t}^{\circ}: 33.1$; Elasticity: 41.

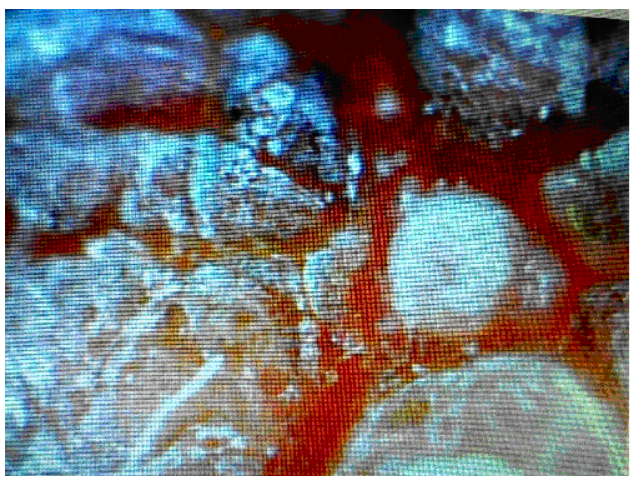

Figure 4. CRACKING NIPPLES: appear as a paved skin with heavy, bleeding crossing stripes. Hydration: $34 ; \mathrm{pH}: 4.6 ; \mathrm{t}^{\circ}$ : 33.4; Elasticity: 42.

\section{Discussion}

Nipple trauma associated with breastfeeding onset is commonplace and mothers cite it as one of the main reasons for pain, cracked or bleeding nipples, and early cessation of breastfeeding [5,6]. Discomfort becomes particularly pronounced on the first post-partum days as breasts fill to the engorgement stage of lactogenesis [17]. Preventing and treating traumatized, sore nipples quickly and effectively is crucial to ensure that the bonding experience is a pleasant one and to encourage mothers to continue in the face of further complications and difficulties $[8,18]$.

A variety of interventions have been used to prevent or to treat nipple trauma and/or pain associated with breastfeeding. However, the mechanisms of action are not entirely clear [19].These can include 
topical creams, solutions or sprays, restricting the infant's time at the breast, exposure of the nipples to dry heat or ultraviolet light and air-drying, hardening of the nipple skin, and pre- or post-natal breastfeeding education [8]. None of these measures appear to be particularly efficacious nor have they been investigated scientifically. For the firs time, we chose to adopt a reproducible method to measure breast skin barrier early after delivery, evaluating the hydration and $\mathrm{pH}$ values with objective observation of dermatological features of nippleareola complex in early post partum breastfeeding, a crucial time for breastfeeding establishment. This bioengineering method represents a new opportunity to improve breast care and breastfeeding success, making breastfeeding mothers aware of their personal dermatological breast hydration and $\mathrm{pH}$ characteristics and informed of results of adopted breast care strategies to prevent cracked nipples.

It is well known that skin barrier function, which represents protection from water loss from the body, depends on several factors including the level of hydration, the cellular properties of the corneocytes, the association and turnover of these cells in the stratum corneum, the amount and composition of intercellular lipids, and skin surface $\mathrm{pH}$ (a protective acid mantle) [12]. This is relevant, since the water content of the stratum corneum and skin surface lipids form a balance, very important for breast skin function and integrity may be now correctly monitored [20].

Even if there aren't previous practices about the use of macro photography for diagnostic approach of the NAC hydration in breastfeeding women, both medical-obstetric staff and mothers easily realize a low hydration and cracking nipples; in fact, they are evident for their red hematic colour paved skin that reveals breast pain and bleeding. Nevertheless, thanks to the photographic image that evolves over time, obstetricians and new mothers could make an effort into improving skin barrier and breastfeeding practices.

\section{Conclusion}

In conclusion, this non-invasive, rapid, and scientific approach for breast care could be recommended for improve skin barrier and preventing of nipple cracking along with teaching better breastfeeding technique at the initiation of breastfeeding. Further long-term studies are warranted to confirm the beneficial effects on areola skin barrier of macro photography determination in a very sensitive patient population.

\section{References}

1. Schaal B, Doucet S, Sagot P, Hertling E, Soussignan R (2006) Human breast areolae as scent organs: morphological data and possible involvement in maternal-neonatal coadaptation. Dev Psychobiol 48: 100-110. [Crossref]
2. Zanardo V, Giarrizzo D (2015) Re. "Review on emu products for use as complementary and alternative medicine". Nutrition 31: 415. [Crossref]

3. Varendi H, Porter RH, Winberg J (1994) Does the newborn baby find the nipple by smell? Lancet 344: 989-990. [Crossref]

4. Delaunay-El Allam M, Marlier L, Schaal B (2006) Learning at the breast: preference formation for an artificial scent and its attraction against the odor of maternal milk. Infant Behav Dev 29: 308-321. [Crossref]

5. Tait $\mathrm{P}$ (2000) Nipple pain in breastfeeding women: causes, treatment, and prevention strategies. J Midwifery Womens Health 45: 212-215. [Crossref]

6. Cable B, Stewart M, Davis J (1997) Nipple wound care: a new approach to an old problem. J Hum Lact 13: 313-318. [Crossref]

7. Minchin MK (1989) Positioning for breastfeeding. Birth 16: 67-73. [Crossref]

8. Lochner JE, Livingston CJ, Judkins DZ (2009) Clinical inquiries: Which interventions are best for alleviating nipple pain in nursing mothers? J Fam Pract 58: 612a-612c. [Crossref]

9. Foxman B, D'Arcy H, Gillespie B, Bobo JK, Schwartz K (2002) Lactation mastitis: occurrence and medical management among 946 breastfeeding women in the United States. Am J Epidemiol 155: 103-114. [Crossref]

10. Seidenari S, Giusti G (1995) Objective assessment of the skin of children affected by atopic dermatitis: a study of $\mathrm{pH}$, capacitance and TEWL in eczematous and clinically uninvolved skin. Acta Derm Venereol 75: 429-433. [Crossref]

11. Zemtsov A, Gaddis M, Montalvo-Lugo VM (1996) Moisturizing and cosmetic properties of emu oil: a pilot double blind study. Australas J Dermatol 37: 159-161. [Crossref]

12. Knor T, Meholjiä $†$-FetahoviÄ $†$ A, MehmedagiÄ $†$ A (2011) Stratum corneum hydration and skin surface $\mathrm{pH}$ in patients with atopic dermatitis. Acta Dermatovenerol Croat 19: 242-247. [Crossref]

13. Imokawa G, Akasaki S, Minematsu Y, Kawai M (1989) Importance of intercellular lipids in water-retention properties of the stratum corneum: induction and recovery study of surfactant dry skin. Arch Dermatol Res 281: 45-51. [Crossref]

14. Segre JA (2006) Epidermal barrier formation and recovery in skin disorders. J Clin Invest 116: 1150-1158. [Crossref]

15. Zanardo V, Giarrizzo D, Maiolo L, Straface G (2016) Efficacy of Topical Application of Emu Oil on Areola Skin Barrier in Breastfeeding Women. J Evid Based Complementary Altern Med 21: 10-13. [Crossref]

16. http://www.callegari1930.com

17. de Carvalho M, Robertson S, Klaus MH (1984) Does the duration and frequency of early breastfeeding affect nipple pain? Birth 11: 81-84. [Crossref]

18. Morland-Schultz K, Hill PD (2005) Prevention of and therapies for nipple pain: a systematic review. J Obstet Gynecol Neonatal Nurs 34: 428-437. [Crossref]

19. Zemtsov A, Gaddis M, Montalvo-Lugo VM (1996) Moisturizing and cosmetic properties of emu oil: a pilot double blind study. Australas J Dermatol 37: 159-161. [Crossref]

20. Kim DW, Park JY, Na GY, Lee SJ, Lee WJ (2006) Correlation of clinical features and skin barrier function in adolescent and adult patients with atopic dermatitis. Int $J$ Dermatol 45: 698-701. [Crossref]

Copyright: (C2016 Zanardo V. This is an open-access article distributed under the terms of the Creative Commons Attribution License, which permits unrestricted use, distribution, and reproduction in any medium, provided the original author and source are credited. 